\title{
Combination options with the male urethral sling to treating post-prostatectomy incontinence
}

\author{
Blayne Welk, MD, FRCSC
}

See related article on page 107.

Cite as: Can Urol Assoc J 2011;5(2):112; D01:10.5489/cuaj.11051

$\mathrm{T}$ he male urethral sling (MUS) has been embraced by many urologists as a treatment option for mild to moderate post-prostatectomy incontinence. The potential advantages compared to the artificial urinary sphincter (AUS) make it an attractive option to physicians and patients. However, the published literature on the MUS is limited. The role of a MUS in patients with previous pelvic radiation or more severe incontinence ( $>5$ pads per day) is still unclear.

The authors present a case series of 40 patients treated with a MUS (primarily with the AdVance Sling [American Medical Systems, Minnetonka, MN]). ${ }^{1}$ Their success rate is $63 \%$ (25 of 40 ) and is in keeping with other published series. The authors also present their approach and outcomes for the failed male sling patients (15 of 40), all of whom had a significant initial degree of incontinence (7-10 pads per day) that did not respond to MUS placement. These patients were all treated with a second procedure: either an AUS if they had previous radiation, or activation/implantation of an adjustable continence therapy system (ProACT, Uromedica Inc., Plymouth, MN). After the second procedure, all 15 patients achieved continence ( $\leq 1$ pad per day).

The AdVance MUS is hypothesized to increase the membranous urethral length by elevating the posterior urethra. ${ }^{2}$ This is different from the compressive mechanism of adjustable continence therapy or an AUS. We do not know if the MUS contributed to the continence achieved from the subsequent placement of the adjustable continence ther- apy system or AUS. There were 2 men who had previously failed adjustable continence therapy; they also failed to respond to MUS placement. However, when their ProACT system was reactivated, the combination of the MUS and ProACT resulted in continence. This result suggests that the 2 treatments can contribute to continence in an additive fashion. This concept has also been demonstrated with the successful use of an AdVance MUS in AUS patients with recurrent incontinence. ${ }^{3}$ The combination of a MUS and a compressive device is a novel approach to male patients with persistent or recurrent stress incontinence.

Reconstructive Surgery, Incontinence \& Neurourology Fellow, University of Toronto, Toronto, ON

Competing interests: None declared.

This paper has been peer-reviewed.

\section{References}

1. Al-Najar A, Kaufmann S, Boy S, et al. Management of recurrent post-prostatectomy incontinence after previous failed retrourethral male slings. Can Urol Assoc J 2011;5:107-11; D01:10.5489/cuaj.10092

2. Rehder $P$, Gozzi C. Transobturator Sling Suspension for Male Urinary Incontinence Including Post-Radical Prostatectomy. Eur Urol 2007:52:860-7.

3. Christine B, Knoll LD. Treatment of recurrent urinary incontinence after artificicial urinary sphincter placement using the AdVance male sling. Urology 2010;76:1321-4.

Correspondence: Dr. Blayne Welk, Department of Surgery, Division of Urology, University of Toronto, Sunnybrook Hospital, 2075 Bayview Ave, Rm MG408, Toronto ON M4N 3M5; bkwelk@gmail.com 